\title{
Atmospheric Radar Signal Processing using Complex Wavelet Transforms
}

\author{
D. Leela Rani \\ Dept. of ECE, Sree \\ Vidyanikethan Engineering \\ College,Tirupati.
}

\author{
S. Varadarajan, Ph. D \\ Dept. of ECE, S.V. University \\ College of Engineering, \\ Tirupati.
}

\author{
M. Sadasiva \\ Dept. of ECE, S.V. University \\ College of Engineering, \\ Tirupati.
}

\begin{abstract}
Complex wavelet Analysis have been carried out on backscattered signals of Mesosphere Stratosphere Troposphere (MST) radar, located at Gadanki (13.50N, 79.20E). The echoes received from MST Radar are weak and buried in noise which leads to decrease in signal to Noise Ratio (SNR). Complex wavelet Transforms such as Analytic Wavelet Transform (AWT) and Dual Tree Complex Wavelet Transform (DTCWT) are implemented and are used as denoising algorithms in order to have good improvement in SNR of radar returns even under severe weather conditions. The properties of shift variance associated with good directional selectivity, when compared to discrete wavelet Transform (DWT) forces Complex Wavelet Transforms to be used in the fields of Image Processing and Atmospheric Radar Signal Processing. In addition, DTCWT has Perfect Reconstruction and computational efficiency. Complex wavelet Analysis has been performed on the backscattered signals received in two modes. Results show a good improvement in SNR on application of AWT and DTCWT, when compared to real DWT. The height profiles of SNR for DTCWT are even far better than AWT.
\end{abstract}

\section{Keywords}

MST Radar, Backscattered signal, Analytic Wavelet Transform, Dual Tree Complex Wavelet Transform, Shift invariance, Directional Selectivity.

\section{INTRODUCTION}

Improving SNR, Spectrum cleaning, detection and estimation of desired parameters are the main objectives in Atmospheric Radar Signal Processing. Atmospheric radar signal refers to signal received by MST radar due to backscattering property of atmospheric layers- stratified or turbulent [1]. The received backscattered signals are associated with noise. As the distance between the radar and target increases, the noise dominates the signal which leads to a decrease in signal-tonoise ratio. Improvement in SNR of such signals by reducing noise level is the aim of denoising algorithm [2]. The denoising algorithms used here are implemented using AWT and DTCWT.

Fast Fourier Transform (FFT) is an important tool for analysis and processing of many natural signals. But it is limited to stationary and linear signals. Short Time Fourier Transform (STFT) deals with non-stationary signals but with a trade-off between time and frequency resolutions. Continuous Wavelet Transform is an alternative approach to STFT which overcomes resolution problem by changing the width of window during the computation of transform. As far as, the reconstruction of the signal is concerned, these transform provides highly redundant information, which requires significant amount of computation time and resources.
Discrete Wavelet Transform (DWT) on the other hand, is considerably easier to implement, when compared to Continuous Wavelet Transform and provides sufficient information both for analysis and synthesis of original signal with a significant reduction in computation time. Standard DWT is implemented through a simple filter bank structure and allows Multi Resolution Analysis (MRA). These advantages popularized DWT in many signal processing applications like denoising, Spectrum Cleaning, Evaluation of desired parameters etc. The three main disadvantages associated with DWT are lack of shift invariance, lack of symmetry of mother wavelets and poor directional selectivity. These disadvantages are diminished by the use of complex wavelet transform [3] like Analytic Wavelet Transform (AWT) and Dual Tree Complex Wavelet Transform [3]. In this paper, a simple implementation of AWT and DTCWT with soft thresholding technique is implemented and applied to backscattered radar signal. The height profiles of original signal and signals denoised with AWT, DTCWT are plotted for both vertical and off-vertical directions. Results show a very good improvement in SNR of DTCWT denoised signal.

\section{RADAR DATA SPECIFICATIONS}

The MST Radar is located at Gadanki, near Tirupati $\left(13.47^{\circ} \mathrm{N}\right.$, $\left.79.18^{\circ} \mathrm{E}\right)$. MST radar operates continuously for different type of experimental observations. Here the backscattered signals provided by MST Radar corresponds to experiments in the lower atmosphere; i.e. the region of $3.6 \mathrm{~km}$ to $\sim 25 \mathrm{~km}$. Only sample data is used for analysis to demonstrate the technique. Radar records data for each range gate and the resolution of sample can vary depends on experimental specification. Here the sampling interval corresponds to $150 \mathrm{~m}$ ( 1 micro sec.) in space is used and number of samples taken on each range gate is about 512 points. Radar can be operated in two modes. In first mode, radar echoes are recorded in 6 beam directions, viz. East, West, Zenith-x, Zenith-y, North and South directions whereas in second mode, i.e, vertical mode, the data is recorded in either Zenith-x or Zenith-y directions. These data are complex in nature and hence the method adopted is complex signal analysis. Each channel (I and Q) is independently treated for all preliminary processes and combined in the final stage while computing Complex Wavelet Transforms for one beam.

\section{DISCRETE WAVELET TRANSFORM (DWT)}

The signal is passed through a series of high pass filters to analyze the high frequencies and it is passed through a series of low pass filters to analyze the low frequencies. The low pass and high pass filters used in DWT are called Quadrature Mirror Filters. The resolution of the signal, which is a measure of amount of detail information in the signal, is changed by filtering operations. The scale is changed by sub sampling operation. DWT employs two sets of functions, 
called scaling and wavelet functions, which are associated with low pass and high pass filters respectively. The decomposition of the signal into different frequency bands is simply obtained by successive high pass and low pass filtering of the time domain signal. At each level of decomposition DWT coefficients are obtained. Hence the DWT of original signal is obtained by concatenating all coefficients starting from the last level of decomposition. The DWT will then have the same number of coefficients as the original signal. The implementation of ID DWT is shown in figure 1.

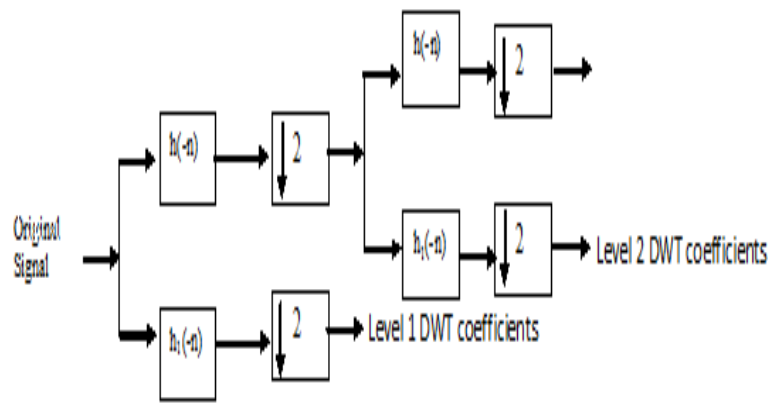

Fig 1: 2-channel, 2-level Discrete Wavelet Transform

DWT offers good time resolution at high frequencies and good frequency resolution at low frequencies. The above procedure is followed in reverse order for reconstruction. Other wavelet transforms which overcomes the disadvantages of DWT are Double Tree Complex Wavelet Transform (DTCWT), Undecimated Wavelet Transform (UDWT), and Analytic wavelet Transform (AWT)

\section{ANALYTIC WAVELET TRANSFORM (AWT)}

The analytic wavelet transform (AWT) is a wavelet transform that provides both the magnitude and phase information of signals in the time-scale or time-frequency domain. The magnitude information returned by AWT describes the envelopes of signals which can be used for time-frequency analysis. The phase information encodes the time related characteristics of signals, for applications such as instantaneous frequency estimation [4].

A pair of real mother wavelets such that one is approximately the Hilbert transform of other is designed. This wavelet pair defines AWT. A complex wavelet coefficient is obtained by interpreting the wavelet coefficient from one DWT tree as being its real part, whereas the corresponding coefficient from other tree is interpreted as its imaginary part [5]. The implementation of AWT is shown in figure 2 .

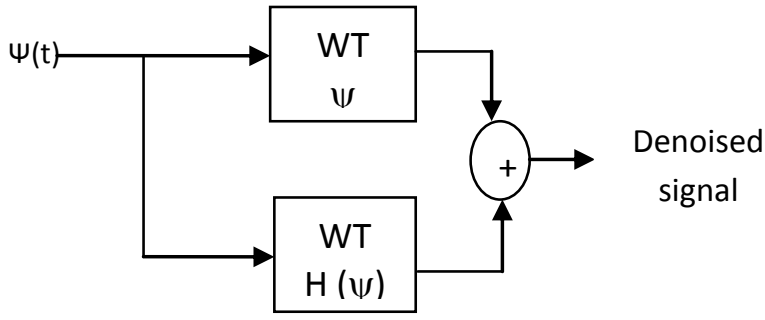

Fig 2: Implementation of Analytic Wavelet Transform
Hilbert transform is first applied to backscattered time series radar data. The wavelet transform is then applied to analytical signal associated with the input radar data.

An analytic wavelet $\psi_{\mathrm{a}}(\mathrm{t})$ is defined as complex-valued wavelet given by

$$
\psi_{\mathrm{a}}(\mathrm{t})=\psi(\mathrm{t})+\mathrm{jH}\{\psi(\mathrm{t})\}
$$

Where $\psi(t)$ denotes real part of complex wavelet and $H\{\psi(t)\}$ is Hilbert transform of real part $\psi(\mathrm{t})$ in the range of $-\infty<\mathrm{t}$ $<\infty$;

$$
\mathrm{H}\{\psi(\mathrm{t})\}=* 1 /(\pi \mathrm{t})
$$

Forming an analytic wavelet in time domain removes the negative frequencies of the spectrum, resulting in an analytic wavelet with one-sided spectrum in frequency domain. The wavelet transform is by nature convolution of the signal being analyzed with the mother wavelet function in the time domain i.e., the computation of forward wavelet transforms and filtering of wavelet coefficients is done. Then the filtering coefficients are obtained by the application of inverse wavelet transform. The advantage of the analytic wavelet coefficients is that their envelopes can be readily extracted from their real and imaginary parts.

\section{DUAL TREE COMPLEX WAVELET TRANSFORM}

To overcome the disadvantages of DWT, Kingsbury [6] implemented dual-tree wavelet transform with excellent directionality, reduced shift sensitivity and explicit phase information. To achieve approximate shift invariance with real DWT, the sampling rate is doubled at each level of tree. Figure 1 shows the tree. To make this possible, the samples must be evenly spaced. The sampling rates in tree a of figure 3 can be doubled by eliminating the down-sampling by 2 after the level 1 filters, H0a and H1a

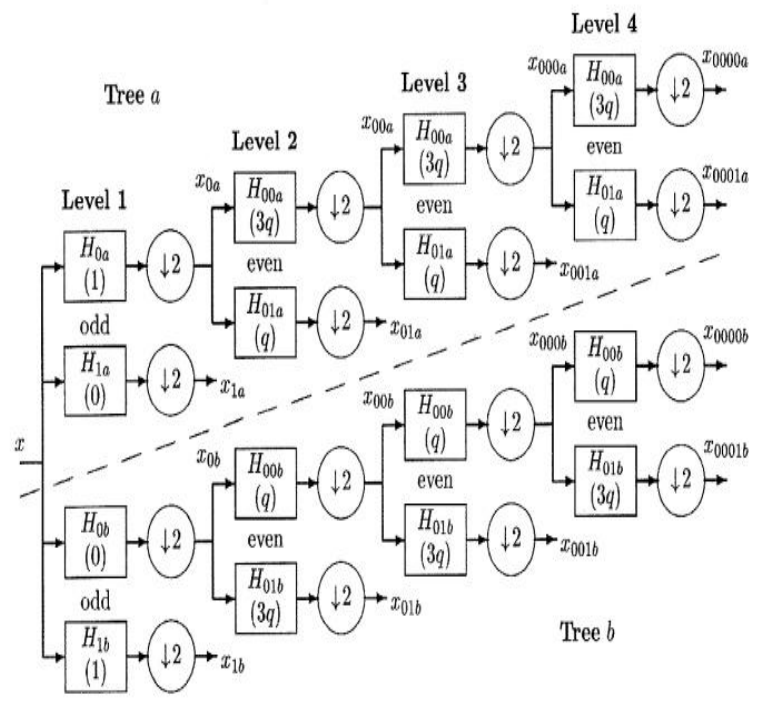

Fig 3: Q-Shift version of the Dual Tree Complex Wavelet Transform

This is equivalent to two parallel fully-decimated trees, a and $\mathrm{b}$, provided that the delays of $\mathrm{HOb}$ and $\mathrm{H} 1 \mathrm{~b}$ are one sample offset from H0a and H1a. This offset ensures the pickup of opposite samples in both trees. To get uniform intervals between samples from the two trees below level 1, the filters in one tree must provide delays that are half a sample different 
(at the filter input rate) from those in the other tree. This statement is also supported by Selesnick [7].

It consists of a pair of filter banks that simultaneously operate on the input signal and provide two wavelet decomposition. The filters are designed in such a way that the sub band signals in one filter bank can be interpreted as the real part of a complex wavelet transform and sub band signals of other filter bank can be interpreted as imaginary part i.e., the wavelets associated with the filter banks form Hilbert pair.

The limitation with this implementation is that it is two times expansive because for an N-point signal it gives 2N DWT coefficients, which require more storage space than the input signal. Hence it is redundant.

\subsection{Problems of Odd-even length filter}

Dual Tree DWT is also complex DWT. In each sub band, one tree produces the real part and the other the imaginary part of the Complex wavelet coefficient and so the filters in the two trees cannot be identical. The first implementation proposed had the constraint of linear phase, and to accomplish this, the implementation required odd-length filters in one tree and even-length filters in the other. Greater symmetry between the two trees occurs if each tree uses odd and even filters alternately from level, but this is not essential. The problems with the odd/even filter approach to achieve this delay are

- The sub-sampling structure is not symmetrical.

- The two trees have a slightly different frequency responses.

- The filter sets must be bi-orthogonal because they are linear phase.

These drawbacks have been overcome with a more recent form of the dual tree known as a Q-shift dual tree.

\subsection{Q-Shift filters}

Two sets of filters are used. The filters at level 1 , and the filters at all higher levels. The filters beyond level 1 have even length but are no longer strictly linear phase. Instead they are designed to have a group delay of approximately $1 / 4$ sample (q). The required delay difference of $1 / 2$ sample (2q) is then achieved by using the time reverse of the tree a filters in tree $\mathrm{b}$, So that, the delay of tree-b filters become $3 \mathrm{q}$.

The filter coefficients are no longer symmetric hence it is possible to design the Perfect Reconstruction (PR) filters that are orthonormal, so that the synthesis filters are just the time reverse of the equivalent analysis filters in both trees. Hence all filters beyond level 1 are derived from the same orthonormal prototype set. There are a number of choices of possible filter combinations. The key to design filters for Qshift version of DTCWT is based on selection of a good even length low pass filter with a delay of $1 / 4$ sample which also satisfies the standard orthonormal PR condition of 2-band filter bank. The Q-shift transform retains the good shift invariance and directionality properties while also improving the sampling structure.

\section{RESULTS AND DISCUSSIONS}

The denoising algorithms of complex wavelets transforms, AWT and DTCWT are simple to implement and requires less computation as redundancy in DTCWT is minimized by the use of simple separable filters. These algorithms are applied on the radar data collected in two different modes.
The data of 07 August 2008 is collected by operating the radar in the first mode i.e., in six directions. The height profiles of SNR for this data are shown in figure 4.

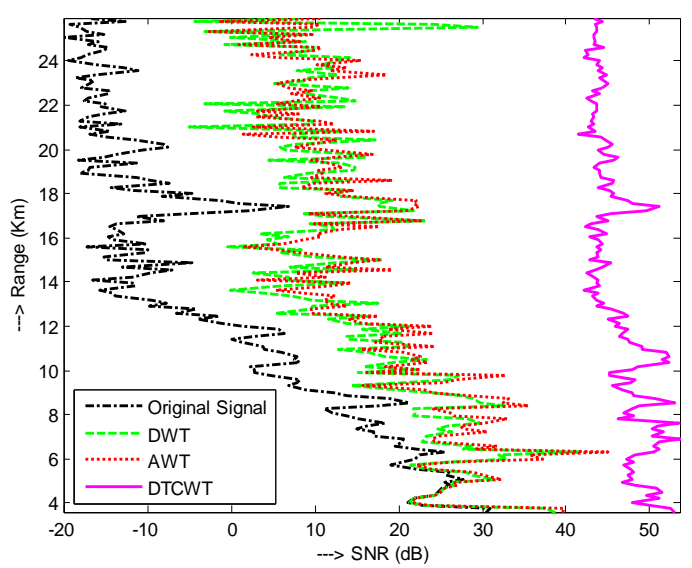

Fig 4: Comparison of Signal-to- Noise Ratio in the first mode

On 18 December 2013, the radar was operated in second mode i.e., the vertical mode. The height profiles of SNR for this data are shown in figure 5 .

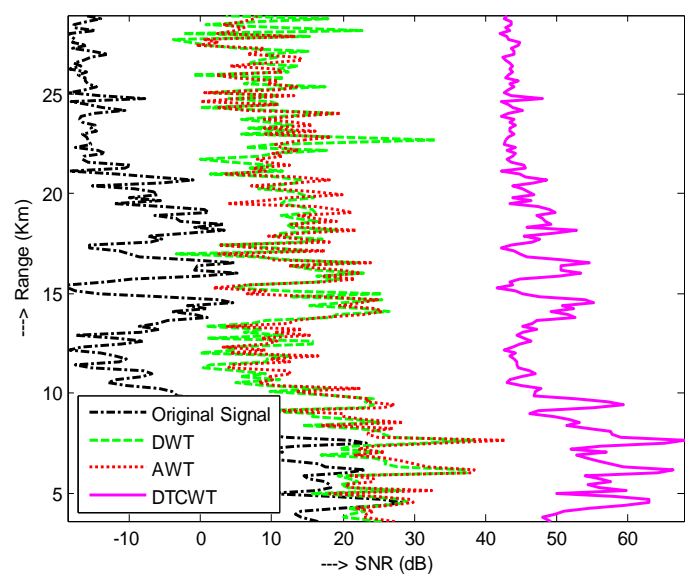

Fig 5: Comparison of Signal-to- Noise Ratio in the second mode

In both the above cases, the profiles are plotted for original (noisy) data and data denoised with DWT, AWT and DTCWT algorithms. Results show very good improvement in the case of DTCWT when compared to DWT and AWT algorithms.

It can be concluded that there is a great improvement in Signal-to-Noise Ratio in the case of DTCWT when compared to the DWT and AWT techniques presented. Good Shift invariance is also achieved with the use of Q-shift filters. Signal-to-Noise Ratio can further be increased by increasing the level of decomposition. 


\section{REFERENCES}

[1] S. Varadarajan, K.Jithendra Reddy and G. Ramachandra Reddy, "Wind profile detection of atmospheric radar signals using wavelets and harmonic decomposition techniques," atmos.sci. Lett.,Vol 5, no.7, pp152-161, Dec2004 Computer Science and Applications. (ISSN: 2229-4597), Vol. 3, No. 6, pp. 29 - 36.

[2] Sreenivasulu Reddy Thatiparthi, Ramachandra Reddy, Gudheti, Varadarajan Sourirajan, "MST Radar signal processing using wavelet-based denoising," IEEE Trans. Geosci. Remote Sens., vol.6, no.4, pp752-756, Oct 2009.

[3] N.G. Kingsbury, "Complex wavelets for shift invariant analysis and filtering of signals," Journal of Applied and Computational Harmonic Analysis, vol. 10, no. 3, pp. 234-253, May 2001.

[4] I.Adam, C.Nafornita, J-M Boucher,A.Isar, "A New Implementation of Hyperanalytic Wavelet Transform ,Proc. Of IEEE Symposium ISSCS 2007, Iasi, Romania,'07,401-404.
[5] D. Sreenivasulu Reddy et al, "A New Image Denoising Scheme using Hyperanalytic Wavelet Transfrom with an Adaptive Wiener Filter", DIGITECH: An International Journal of Computer Science and Applications. (ISSN: 2229-4597), Vol. 3, No. 6, pp. 2936.

[6] N. G. Kingsbury, "The dual-tree complex wavelet transform: A new technique for shift invariance and directional filters," in Proc. Eighth IEEE DSP Workshop, Salt Lake City, UT, Aug. 9-12, 1998.

[7] I. W. Selesnick, R. G. Baraniuk, and N. G. Kingsbury, "The dual-tree complex wavelet transform," IEEE Signal Process. Mag., vol. 22, no.6, pp. 123-151, Nov. 2005.

[8] D. Leela Rani, S.Varadarajan, M.Saasiva, "MST Radar Signal Processing using Analytic Wavelet Transform," Proc., Second International Conference on Advanced Computing Methodologies,(ISBN:9789351071495), GRIET, Hyderabad, pp. 353-355,21-23 November 2013. 